\title{
Introducción del sistema europeo de transferencia de créditos (ECTS) en la Facultad de Medicina de La Laguna
}

\author{
Introduction of the European Credit Transfer System (ECTS) in the medical \\ school of the University of La Laguna
}

Eduardo Doménech Martínez, Luis Hernández Nieto, Luis Morcillo Herrera, Norberto Hernández Siverio y Juan Luis Gómez Sirvent

Facultad de Medicina. Universidad de la Laguna. La Laguna (S/C de Tenerife)

Para la convergencia europea se deben modificar los planes de estudios de las facultades de medicina de forma que el Sistema de Transferencia de Créditos Europeo (ECTS) esté incorporado a los nuevos planes del estudio antes del 2010

Objetivos: Experimentar la introducción del ECTS en el segundo cuatrimestre de quinto curso, modificando la metodología docente.

Diseño del estudio: Se adaptaron los programas teóricos y prácticos y se establecieron unos objetivos docentes y un sistema de evaluación para las asignaturas de Patología Médica de la Sangre y de la Hematopoyesis, de las Enfermedades Infecciosas y del Sistema Endocrino y Nutrición, Pediatría y Patología Quirúrgica de Cara, Cuello, Mama y Glándulas Endocrinas. Se realizaron encuestas a los alumnos en el transcurso de cuatrimestre, utilizando para las respuestas una escala de tipo Likert con cinco grados. Se analizó la priorización de las competencias genéricas y específicas de los alumnos. Para el análisis de los resultados se aplicaron pruebas no paramétricas. Resultados: Los 37 créditos actuales se transformaron
Correspondencia:

E. Doménech Martínez

Teléfono: 922319308

Fax: 922678850

e-mail: edomenec@ull.es en 29.5 ECTS, con 736.5 horas de trabajo para los alumnos. Se redujeron los programas teóricos de 186 lecciones en total a 134 (reducción del $27.96 \%$ ). Los alumnos prepararon y presentaron 58 casos clínicos bajo la supervisión de sus tutores. Los profesores consideraron que el aprendizaje teórico había sido similar al de cursos anteriores (66.6 \%) y el práctico había sido mejor (73.3 \%). El $73.3 \%$ de los alumnos contestaron que el proyecto debía mejorarse, mediante la reducción de la carga de trabajo de los alumnos y mejora del sistema de evaluación. Los alumnos apreciaron de forma estadísticamente significativa que mejoró la docencia práctica y su capacidad para preparar y presentar casos clínicos. El rendimiento académico de los alumnos fue significativamente mejor que el de los alumnos del curso anterior. Los estudiantes estuvieron de acuerdo con la prioridad de competencias de la CND para 9/9 de las generales y 4/17 de las específicas.

Conclusiones: La introducción experimental del ECTS mejoró el rendimiento académico, la formación práctica y el autoaprendizaje de los alumnos. La experiencia fue satisfactoria para profesores y alumnos. La estimación de la carga de trabajo de los alumnos fue subestimada.

Palabras clave: Medicina, enseñanza, ECTS, reforma plan

de estudios, evaluación, estudiantes

Introduction: To comply with the requirements of European convergence, faculties of medicine must modify their curricula and incorporate the European Credit Transfer System (ECTS) before 2010. 
Objectives: To test the introduction of ECTS in the 2nd term of the 5th year medicine course and the appropriately modified teaching methodology. Study design: Theoretical and practical programs were adapted; academic objectives and student evaluation system were established for the following subjects: medical pathology of blood and haematopoiesis, infectious diseases and endocrine system and nutrition, paediatrics and surgical pathology of the face, neck, breast and endocrine glands. Students were surveyed during the term, using a 5-point Likert-type scale. We recorded student priorities for general and specific parameters, and the results were analyzed using non-parametric tests.

Results: Thirty-seven credits were converted into 29.5 ECTS credits, representing 736.5 student working hours. The theory program was reduced from 186 to 134 lectures (a reduction of $28 \%$ ). Students presented 58 tutor-supervised clinical cases. The teaching staff considered that learning of theory was similar to previous years $(66.6 \%)$ but that practical learning improved (73.3\%). Most students (73.2\%) thought the program should be improved, especially the examination system, and the workload reduced. Students reported a statistically significant improvement in practical teaching and in their capacity to present clinical cases. Academic performance was significantly higher than that of the 5th year students the previous year. Students agreed with the National Deans Conference priorities for the 9/9 general and $4 / 17$ specific competences.

Conclusions: Introducing ECTS improved academic performance, practical training and self-learning. The project proved satisfactory for staff and students. Student workload was underestimated in the new plan.

Key words: Medicine, teaching, ECTS, Curriculum reform, examination, students

\section{INTRODUCCIÓN}

Como es sabido se ha iniciado un proceso de implantación del Espacio Europeo de Educación Superior (EEES) $)^{1-4}$ y antes del año 2010 deben de estar elaborados los nuevos planes de estudios para la convergencia europea, que deben incorporar el sistema de transferencia de créditos europeo (ECTS).
En nuestro país se publicó en el año 2003 el real decreto que regula el crédito ECTS $^{5}$. Debe ser este un sistema entendible y comparable que mida el trabajo realizado por el alumno, mediante acumulación de créditos realizados que permita la transferencia de los mismos a cualquier universidad europea que participe en este programa. Pero introduce también un matiz importante, como es que la docencia se centra preferentemente en el aprendizaje del alumno y menos en las enseñanzas impartidas por el profesor. El real decreto establece que 1 crédito ECTS equivale a 25-30 horas de trabajo del estudiante. Un curso académico debe tener 60 créditos ECTS y ello supone un trabajo del estudiante de 1.500-1.800 horas anuales.

La reciente actualización de la Directiva Europea relativa al "Reconocimiento de Cualificaciones Profesionales" considera la formación básica del médico en al menos 6 años de estudios, así como las condiciones mínimas de formación (conocimientos y competencias). Recientemente se ha publicado el real decreto que establece la estructura de las enseñanzas universitarias y regula los estudios universitarios oficiales de Grado ${ }^{7}$.

Independientemente de consideraciones críticas, este proceso hay que considerarlo como una nueva oportunidad para llevar a cabo una profunda reforma del sistema de enseñanza universitaria ${ }^{1}$ y no debe quedarse sólo en una mera transformación matemática de la cuantificación de los créditos, sino que debe ir vinculado a un cambio en el modelo docente, debiendo transformarse el profesor instructor del actual sistema en el profesor tutor del nuevo sistema de enseñanza y el currículum debe basarse en competencias, si entendemos por competencias el conjunto de conocimientos, habilidades y actitudes que debe poseer el alumno al finalizar el periodo formativo ${ }^{1,8,9,10}$. Las competencias deben desarrollarse en contenidos comunes que afecten a la totalidad de los centros ${ }^{8,11}$. Las directrices generales que se establezcan por el Gobierno deben ser flexibles con el fin de que cada Facultad pueda seleccionar el modelo educacional que se considere más adecuado en su contexto (infraestructuras, recursos humanos, situación de partida, etc. $)^{1,11}$.

La Conferencia Nacional de Decanos (CND) de Facultades de Medicina ha procedido a la identificación y delimitación de las competencias finales del futuro profesional médico, tras haber realizado 1077 encuestas, de las cuales un $38.71 \%$ corresponden a profesores permanentes de universidad, un $26.27 \%$ a profesores contratados, un $16.15 \%$ a médicos resi- 
dentes, un $6.87 \%$ a gestores sanitarios y un $11.97 \%$ a médicos sin vinculación con la universidad ${ }^{11}$. Estas competencias son genéricas o transversales para un licenciado (instrumentales, personales y sistémicas) que han sido tomadas del Proyecto Tuning ${ }^{8}$, y específicas de la formación disciplinar y profesional del médico general y para ello se han inspirado en los requisitos globales mínimos esenciales en Educación Médica del Instituto para la Educación Médica Internacional ${ }^{12}$.

El objetivo fundamental de este trabajo es llevar a cabo un estudio piloto basado en la introducción del sistema ECTS en una serie de asignaturas de forma independiente respecto al conjunto del plan de estudios, modificando para ello la metodología docente tradicional. Buscamos ajustar las cargas de trabajo de los alumnos en las diferentes materias con los créditos ECTS y mejorar su formación. Nos propusimos también comparar la identificación de competencias genéricas y específicas de nuestros alumnos de quinto curso respecto a la efectuada por profesionales sanitarios ${ }^{11}$. Consideramos que la experiencia obtenida podría ser de utilidad para el diseño de la reforma del plan de estudios de Medicina en lo que respecta a las asignaturas clínicas.

\section{MATERIAL Y MÉTODOS}

El diseño del estudio consistió en transformar los créditos actuales en créditos ECTS, adaptando los programas teóricos y la docencia práctica, tras establecer unos objetivos docentes, un sistema de evaluación de los alumnos y del propio proyecto piloto.

La experimentación se llevó a cabo de Febrero a Julio del 2005, en el segundo cuatrimestre de quinto curso. Durante dicho cuatrimestre se imparten las asignaturas troncales siguientes "Patología Médica de la Sangre y de la Hematopoyesis" (HEMATOL) con 6 créditos (2.5 teóricos y 3.5 prácticos), "Patología Médica de las Enfermedades Infecciosas" (INFEC) con 6 créditos (2.5 teóricos y 3.5 prácticos), "Patología Médica del Sistema Endocrino y Nutrición" (ENDOCRINO) con 6 créditos (2.5 teóricos y 3.5 prácticos), "Patología Quirúrgica de cara, cuello, mama y glándulas endocrinas" (PATOLQUIR) con 4.5 créditos (2 teóricos y 2.5 prácticos) y "Pediatría" con 15 créditos (8 teóricos y 7 prácticos). Participaron en el proyecto piloto los alumnos no repetidores (84/112) matriculados en dicho cuatrimestre.

Para la transformación del total de horas asignadas a una materia en créditos ECTS, se siguieron las directrices para los proyectos pilotos del
Vicerrectorado de Planes de Estudio y Titulaciones de nuestra universidad ${ }^{13}$, que fueron adaptadas del informe técnico de la CRUE ${ }^{14}$. Utilizamos el método impositivo para la asignación de horas a las diferentes actividades, por ser el que se recomienda inicialmente dado que es el mas sencillo ${ }^{15}$ : cada hora de teoría debía ser computada como una hora de trabajo del alumno más una hora y media de estudio para asimilación y preparación para evaluación, y cada hora de práctica clínica supondría media hora más de estudio del alumno. Para las prácticas de laboratorio con simuladores el alumno precisaría por cada hora de práctica otra hora de trabajo; por cada hora de seminario/caso clínico expuesto, el alumno precisaba al menos otra media hora para asimilación y estudio, y cada media hora de tutoría le supondría 1.5 horas de trabajo adicional para realizar las tareas encargadas por el tutor.

Los fundamentos científicos y las habilidades que debían adquirir los estudiantes en las asignaturas cursadas, se establecieron siguiendo los modelos propuestos en el Anexo II del Libro Blanco del Grado de Medicina de la ANECA $^{11}$. Para conocer los fundamentos científicos de las asignaturas se elaboraron los programas teóricos y se fijó un calendario en el que quedaba recogido el día y el profesor que impartiría cada lección. Se redujeron los programas teóricos de 186 lecciones en total a 134 (reducción del $27.96 \%$ ). Pediatría pasó de 80 a 52 lecciones, ENDOCRINO de 35 a 28, HEMATOL de 30 a 20, INFEC de 29 a 20, en PATOLQUIR de 22 a 14. El número de horas de lecciones magistrales a la semana pasó de 13.75 a 8.5 horas. Para adquirir las habilidades clínicas los alumnos debieron realizar el programa de prácticas y se proporcionaba a cada alumno una ficha con los objetivos que debía alcanzar, lo que a su vez debería certificar su profesor de prácticas. Cada alumno tenía cuatro semanas de prácticas para cada uno de los bloques de prácticas: Pediatría, Patología Médica y Patología Quirúrgica. Se elaboraron unos objetivos comunes para las prácticas de las tres asignaturas de Patología Médica.

Para contribuir a lograr los objetivos instrumentales, personales y sistémicos generales y las competencias específicas en lo referente a valores profesionales, actitudes, comportamientos y ética, desarrollar las habilidades de comunicación, el manejo de la información médica y el desarrollo del análisis critico e investigación, los alumnos distribuidos en subgrupos de 3-4, tuvieron que preparar al menos un caso clínico bajo la supervisión de su tutor Los alumnos prepararon y presentaron al conjunto de alum- 
nos 58 casos clínicos: Pediatría 14, ENDOCRINO 8, HEMATOL 16, INFEC 12 y PATOLQUIR 8, cuyo enunciado se hizo público al inicio del cuatrimestre. Se introdujeron en la programación 5.5 horas a la semana para impartir los seminarios y casos clínicos (Pediatría 2 horas, para las asignaturas de Patología Médica 3 horas y para la Patología Quirúrgica media hora a la semana).

La programación de actividades se realizó con arreglo al esquema que figura en la Tabla 1, en la que puede observarse que los 37.5 créditos de las asignaturas se transformaron en 29.5 créditos ECTS, con una estimación de 736.5 horas de trabajo del alumno, para lo que se utilizó una equivalencia de 1 ECTS corresponde a 25 horas de trabajo del alumno. Se optó por incluir un horario de tutorías dentro del esquema de prácticas, asignando a cada subgrupo de 4-10 alumnos un profesor tutor.

La calificación de los alumnos se planificó de la siguiente forma: a) La evaluación continuada por el tutor equivaldría al $30 \%$ de la nota final, para lo que se confeccionó un portafolio con los datos de cada alumno, en el que figuraba las fechas de las entrevistas, los problemas planteados, las tareas encomendadas y los trabajos realizados, los conocimientos mostrados y la preparación y presentación del caso clínico. b) La evaluación de las prácticas com- portaría el $30 \%$ de la nota final, utilizando la ficha en la que se consignaban por el profesor de prácticas la asistencia y las habilidades clínicas del alumno, su actitud y aptitud. c) La evaluación de las enseñanzas teóricas mediante examen de preguntas de respuesta múltiple equivaldría al $40 \%$ de la nota final. En algunas asignaturas como en INFECC y ENDOCRINO hubo que hacer adaptaciones dado que sólo había 1 y 2 profesores asociados clínicos respectivamente, y la evaluación final del alumno se compuso de un $60 \%$ por el examen teórico, un $20 \%$ por la nota de prácticas y un $20 \%$ de la nota correspondiente a la participación en los seminarios y exposición de casos clínicos.

Antes del inicio del segundo cuatrimestre se informó adecuadamente a los alumnos sobre el proyecto, se introdujo el mismo en la página Web de nuestra Facultad (www.medicina.ull.es), y a petición de los alumnos a mediados del cuatrimestre se realizaron aclaraciones adicionales.

Para la evaluación del proyecto se realizaron tres encuestas a los alumnos: al inicio del segundo cuatrimestre del curso 2004-05, antes de realizar el primer examen teórico final y al finalizar el curso académico en la Facultad de Medicina, utilizando para las respuestas una escala tipo Likert con cinco grados ${ }^{16,17}$. La encuesta constaba de 15 preguntas, de forma que

TABLA 1. Esquema de la transformación de créditos ECTS en Medicina, con la distribución de las horas para las actividades de los alumnos.

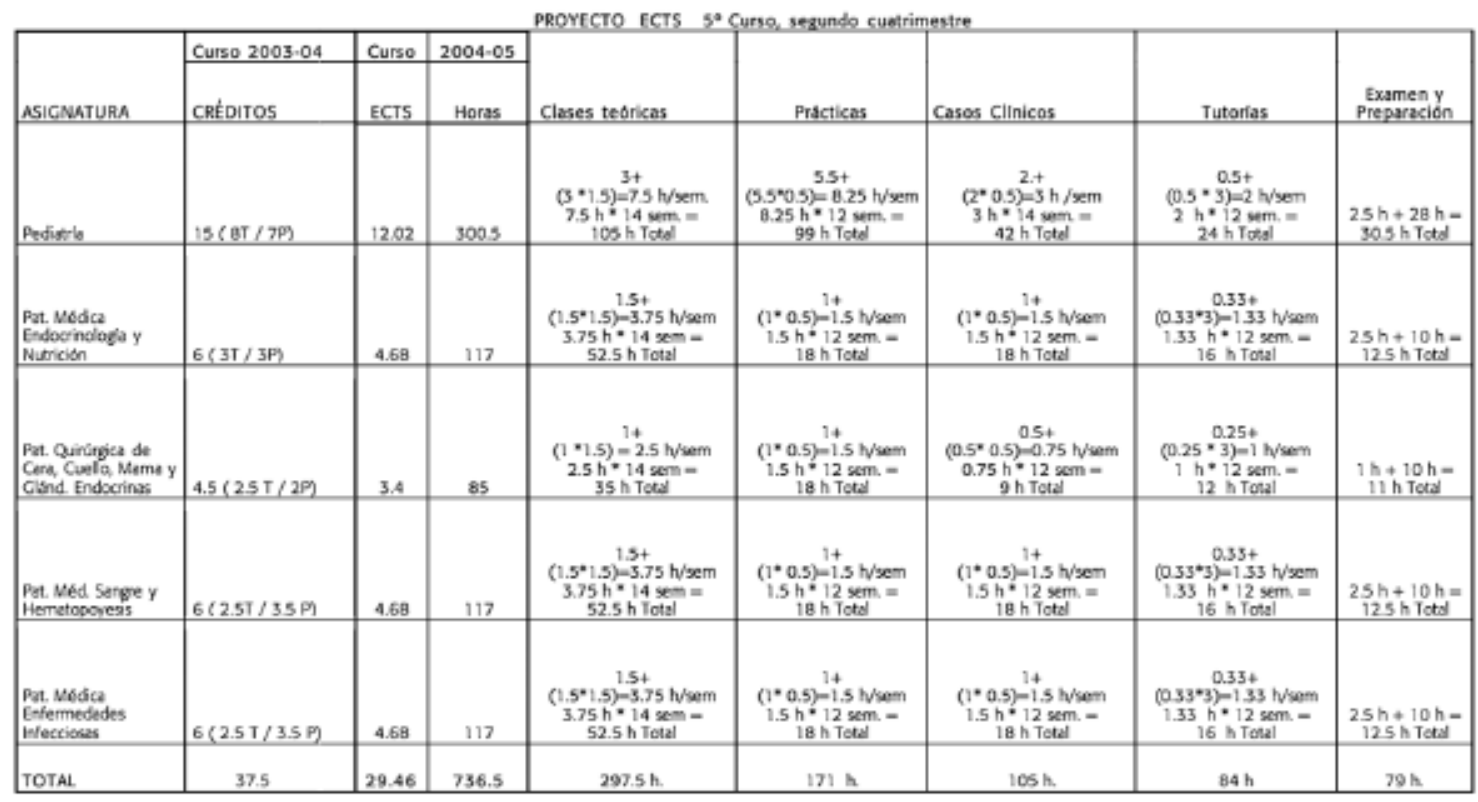


Tabla 2 Encuesta realizada a los alumnos de Medicina para la valoración de la docencia en nuestra Facultad antes y después de la introducción de los créditos ECTS

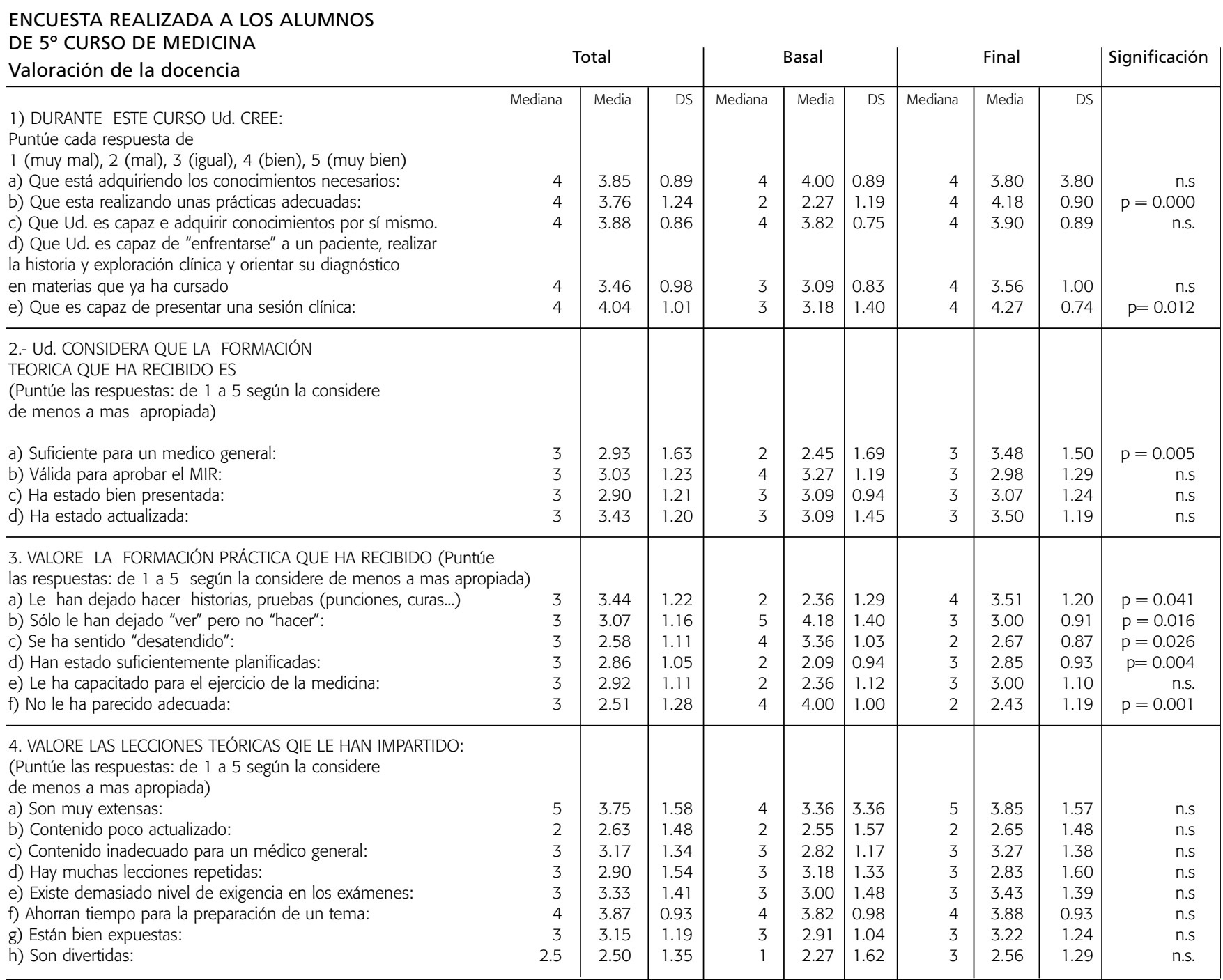

El número de respuestas de la encuesta basal fue de 31 (al comienzo del cuatrimestre) y de 48 para la encuesta final (al terminar el curso).

las preguntas 1, 2, 3 y 4 (23 ítems, Tabla 2) evaluaban la docencia en nuestra Facultad de Medicina. Las preguntas 5,6 y 7 hacían referencia a las competencias generales (23 ítems) y las preguntas 8, 9, 10, 11 y 12 a las competencias específicas (33 ítems), utilizando el modelo de encuesta que había elaborado la $\mathrm{CND}^{11}$. Las preguntas 13 y 14 (21 ítems, Tabla 3 ) valoraban el proyecto de innovación docente y estimaban las horas de trabajo del alumno. La pregunta 15 era de tipo abierto para que los alumnos expusieran las razones por las que debía o no seguir el proyecto. La pregunta 16 se refería a las asignaturas optativas y créditos de libre elección.

El análisis estadístico de las respuestas a las preguntas de las encuestas se realizó con el paquete estadístico SPSS 12 para Windows, aplicando las pruebas no paramétricas de la U de Mann-Whitnell para ver si existían diferencias estadísticas con el transcurso del proyecto. También se utilizó la prueba de la $\mathrm{Chi}^{2}$. Se consideró que existía significación estadística cuando el valor de la "p" era menor de 0.05.

\section{RESULTADOS}

\section{a) Valoración por los profesores de la introducción de los créditos ECTS}

En la encuesta realizada al profesorado para valorar la experiencia del Proyecto, el $66.6 \%$ consideraron que la formación teórica de los alumnos había sido similar al de cursos anteriores y respecto a su 
Figura 1. Mejora del rendimiento académico comparando las calificaciones obtenidas por los alumnos en la convocatoria de Junio de los cursos 2003.04 y 2004-05.

\section{CALIFICACIONES EN JUNIO}

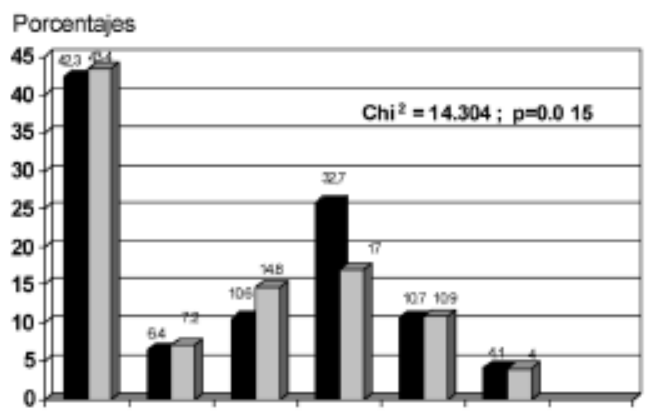

de la docencia en nuestra Facultad, que se realizó al inicio del cuatrimestre y al finalizar el curso.

La estimación de las horas de trabajo del alumno queda recogida en la Tabla 3 (contestaron el $64 \%$ de los encuestados). Respecto a las horas necesarias para la preparación de exámenes la estimación fue de 196.7 \pm 90.8 horas, mientras que los autores del proyecto habíamos calculado 28 horas (Tabla 1). Para realizar el trabajo derivado de las prácticas los alumnos estimaron 25.3 horas a la semana, mientras que nosotros habíamos calculado 6.8 horas a la semana. Para la preparación de los casos clínicos los alumnos estimaron 16.3 horas y nosotros 8.3 horas a la semana. Los alumnos calcularon que debían estudiar 34 horas a la semana (Tabla 3) y nosotros habíamos estimado sólo 12.75 horas (Tabla 1). Para los cálculos de las horas de trabajo derivado de las prácticas y el de las horas para preparación de casos clínicos por los alumnos, se dividió por 3 la suma de todas las asignaturas, dado que los alumnos se distribuían en 3 bloques de prácticas que no eran coincidentes en el tiempo.

En cuanto a las asignaturas optativas y de libre elección, los alumnos fueron casi unánimes al valorar negativamente estas asignaturas (el $89.9 \%$ contestaron que debería disminuirse la carga docente de las mismas).

Respecto a la evaluación global de la experiencia si bien sólo un $25.2 \%$ de los alumnos le dieron la máxima puntuación, cuando se les pidió en la última encuesta que evaluaran cada una de las asignaturas las puntuaciones fueron en todos los casos superiores al "aprobado": en una escala de 1 a 5 éstas fueron: INFEC $3.57 \pm 0.99$, Pediatría $4.02 \pm 1.28$, PATOLQUIR $3.05 \pm 1.38$, HEMATOL $3.65 \pm 0.96$ y ENDOCRINO $3.60 \pm 1.07$.

En la pregunta de expresión libre y opcional sobre si debía o no continuar la experiencia en próximos cursos, el $65.7 \%$ contestó inicialmente (antes del primer examen) que sí, pero dicho porcentaje se elevó al $78.1 \%$ al finalizar el curso, con un valor promedio del 73.2\%. Las razones argüidas mayoritariamente para que continuara el proyecto fueron que las prácticas habían sido mejores, y que les parecían muy útiles los seminarios y los casos clínicos. Pero un $73.3 \%$ también contestaron que debía mejorarse el proyecto, mediante la disminución de la carga de trabajo de los alumnos (98.8\% de los que contestaron la encuesta), y la mejora del sistema de evaluación (88.2\% de las respuestas).

\section{b) Valoración por los alumnos de la docencia en nuestra Facultad y de la introducción de los créditos ECTS}

En la Tabla 2 quedan recogidos los resultados de la encuesta respecto a la valoración por los alumnos

\section{c) Priorización por los alumnos} de las competencias genéricas y especificas En la Tabla 4 se muestran las respuestas de los 
Tabla 3 Encuesta realizada a los alumnos de Medicina para valorar el proyecto de innovación docente y estimar las horas de trabajo del alumnado

ENCUESTA REALIZADA A LOS ALUMNOS

DE $5^{\circ}$ CURSO DE MEDICINA

Valoración de la docencia

\begin{tabular}{|c|c|c|c|c|c|c|c|c|c|}
\hline \multicolumn{3}{|c|}{ Total } & \multicolumn{3}{|c|}{ Basal } & \multicolumn{3}{|c|}{ Final } & \multirow[t]{2}{*}{ Significación } \\
\hline Mediana & Media & DS & Mediana & Media & DS & Mediana & Media & DS & \\
\hline 4 & 3.72 & 0.82 & 4 & 3.68 & 0.93 & 4 & 3.58 & 0.82 & n.s \\
\hline 4 & 3.82 & 0.77 & 4 & 3.83 & 0.73 & 4 & 3.67 & 0.75 & n.s \\
\hline 3 & 2.39 & 0.88 & 2 & 2.20 & 0.86 & 3 & 2.60 & 0.87 & n.s \\
\hline 4 & 3.43 & 1.01 & 4 & 3.37 & 0.93 & 4 & 3.42 & 1.11 & n.s \\
\hline 4 & 3.77 & 1.04 & 4 & 3.91 & 0.91 & 4 & 3.63 & 1.14 & n.s \\
\hline 3 & 3.32 & 0.78 & 3 & 3.37 & 0.93 & 3 & 3.33 & 0.78 & n.s \\
\hline 5 & 4.40 & 0.95 & 5 & 4.50 & 0.78 & 5 & 4.13 & 1.14 & n.s \\
\hline 3 & 3.05 & 1.06 & 3 & 3.07 & 0.99 & 3 & 3.04 & 1.13 & n.s \\
\hline 2 & 2.35 & 1.07 & 2 & 2.28 & 1.07 & 2 & 2.42 & 1.09 & n.s \\
\hline & 196.74 & 90.79 & & 176.30 & 38.85 & & 220.00 & 88.80 & n.s \\
\hline & 98.60 & 46.65 & & & & & 98.60 & 46.65 & \\
\hline & 90.33 & 55.78 & & & & & 90.33 & 55.78 & \\
\hline & 9.80 & 6.04 & & 8.45 & 4.01 & & 11.10 & 7.33 & n.s \\
\hline & 10.69 & 10.5 & & & & & 10.69 & 10.5 & \\
\hline & 6.97 & 3.69 & & & & & 6.97 & 3.69 & \\
\hline & 15.56 & 9.14 & & 13.23 & 6.75 & & 18.15 & 10.76 & n.s \\
\hline & 16.87 & 13.41 & & & & & 16.87 & 13.41 & \\
\hline & 11.74 & 5.9 & & & & & 11.74 & 5.9 & \\
\hline & 8.86 & 6.94 & & 7.57 & 5.48 & & 10.00 & 7.95 & n.s \\
\hline Las & 6.43 & 5.02 & & & & & 6.43 & 5.02 & \\
\hline & 5.92 & 4.65 & & & & & 5.92 & 4.65 & \\
\hline
\end{tabular}

13.- RESPECTO A OTROS CURSOS Y CUATRIMESTRES, COMO VALORARÍA SU EXPERIENCIA COMO ALUMNO DE UN PROYECTO DE INNOVACION DOCENTE DE CREDITOS ECTS Valore cada respuesta de 1 a 5 (de peor a mejor)

a) Las practicas le han parecido

b) La labor del tutor ha sido

c) Las clases teóricas han sido

d) Los seminarios han sido

e) Los casos clínicos expuestos por alumnos

f) El comportamiento de los profesores ha sido

g) El esfuerzo realizado por Ud. ha sido

h) El sistema de evaluación ha sido

i) Los objetivos del proyecto han estado definidos

14) DURANTE ESTE CURSO CON EL PROYECTO DE INNOVACION DOCENTE PARA EXPERIMENTAR CREDITOS ECTS ICUÁNTAS HORAS CALCULA Ud. QUE HA DEDICADO:

a) Para preparar los exámenes finales de Pediatría:

b) Para preparar los exámenes finales de Hematología

c) Para preparar los exámenes finales de Cirugía:

d) Cuantas horas a la semana ha dedicado para realizar las tareas encomendadas en las prácticas de Pediatría

e) Cuantas horas a la semana ha dedicado para realizar las tareas encomendadas en las prácticas de Hematología

f) Cuantas horas a la semana ha dedicado para realizar las tareas

encomendadas en las prácticas de Cirugía

g) Cuantas horas a dedicado en total para preparar los Seminarios

y Casos Clínicos de Pediatría

h) Cuantas horas a dedicado en total para preparar los Seminarios

y Casos Clínicos de Hematología

i) Cuantas horas a dedicado en total para preparar los Seminarios

y Casos Clínicos de Cirugía

j) Cuantas horas a la semana ha dedicado para estudiar

as enseñanzas teóricas de Pediatría

k) Cuantas horas a la semana ha dedicado para estudiar las enseñanzas

teóricas de Hematología

l) Cuantas horas a la semana ha dedicado para estudiar

las enseñanzas teóricas de Cirugía

El número de respuestas de la encuesta basal fue de 31 (al comienzo del cuatrimestre) y de 48 para la encuesta final (al terminar el curso).

alumnos respecto a las priorización de las competencias genéricas y específicas, derivadas de las encuestas que se realizaron al comienzo del cuatrimestre y antes de realizar el primer examen, comparándolas con la priorización realizada por los profesionales médicos en la encuesta de la $\mathrm{CND}^{11}$.

En la figura 2 se muestran aquellas variables que han experimentado una modificación significativa con el desarrollo del proyecto piloto.

\section{DISCUSIÓN}

En las encuestas realizadas se observa claramente que los alumnos percibieron una mejor formación en general, si bien señalan que el esfuerzo realizado fue excesivo y las clases teóricas no alcanzan la valoración promedio de 3. Consideramos que estos resulta- dos se deben a que los alumnos no estaban habituados al trabajo continuado que tuvieron que desarrollar a lo largo del cuatrimestre, y no apreciaron que la evaluación continuada que se les estaba realizando influiría en su calificación final. En experiencias docentes con alumnos de Medicina se ha señalado ${ }^{18,19}$ que aunque los alumnos valoran positivamente la introducción de nuevas metodologías (aprendizaje basado en problemas, portafolio y otros) la mayoría prefieren seguir con el sistema de clases magistrales ${ }^{18}$ y suele haber una reacción adversa inicial que hace aconsejable su introducción de forma voluntaria ${ }^{19}$.

En lo que respecta a las clases teóricas, los profesores, al analizar los resultados de las encuestas de los alumnos, reconocieron su error de programación y la peor calidad de aquellas al haber concentrado excesivamente el contenido de las clases, tras la 
reducción del programa, por lo que se precisaría un mejor ajuste entre el programa teórico reducido y los contenidos de las lecciones.

Entre las razones que daban los alumnos para explicar sus dificultades para realizar las labores encomendadas en las tutorías, señalaban que 2 horas de promedio por la tarde, las tenían ocupadas por las clases de asignaturas optativas y de libre elección. En consonancia con esta apreciación, las respuestas de los alumnos a la encuesta realizada antes de la primera evaluación fueron casi unánimes al valorar negativamente estas asignaturas. Por ello creemos que de realizarse nuevos proyectos de experimentación estos deberían abordar globalmente todas las asignaturas del mismo cuatrimestre, incluidas las optativas y CLE. También compartimos la opinión de los alumnos referente a disminuir la carga lectiva de las asignaturas optativas y CLE.

En lo concerniente a posibles aspectos a mejorar los alumnos señalaron entre otros el sistema de evaluación. Efectivamente, cuando se confrontaron las valoraciones de los distintos tutores, y los criterios utilizados por los dos hospitales universitarios en los que los alumnos realizaron sus prácticas clínicas, se puso de manifiesto que además de cierta subjetividad, diferían las calificaciones entre los hospitales. Resultó difícil la valoración individualizada, dado que los alumnos eran distribuidos en subgrupos de 3-9 (según las asignaturas) y para estimular el trabajo en equipo las tareas se encargaban a cada subgrupo. Aunque era distinta la dedicación de cada alumno para realizar los trabajos encomendados, la preparación de los

Figura 2. Variables que experimentación cambios significativos en las respuestas de los alumnos en las encuestas realizadas a lo largo del curso académico 2004-05.

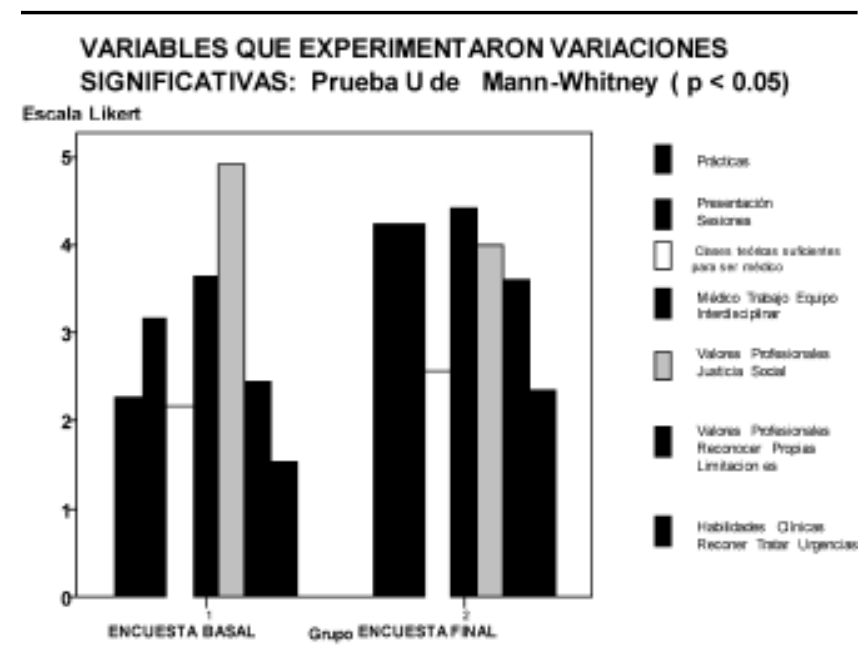

casos clínicos, y sus capacidades de comunicación y conocimientos en la exposición de estos, se tendió a una evaluación similar a los alumnos del subgrupo, lo que perjudicó a los más brillantes. Estas dificultades han sido referidas por otros autore ${ }^{20}, \mathrm{y}$ se ha señalado que la concordancia y discriminación fueron más altas entre los evaluadores más expertos y que se precisa dar más formación a los evaluadores. Por ello para el próximo curso se han programado reuniones para mejorar el sistema de evaluación, y como medida provisional se ha optado por incrementar el peso del examen teórico $(60 \%)$ sobre la nota final.

Los alumnos consideraron que sí habían alcanzado los objetivos, a pesar de que, en su opinión, no habían estado bien expuestos en el proyecto. (Tabla 3). De hecho hubo que realizar a mitad del cuatrimestre una reunión aclaratoria, coincidiendo con la atención a las quejas de que estaban sometidos a una excesiva carga de trabajo. Creemos que la introducción de cambios en los programas y metodologías docentes debe ir precedida de reuniones explicativas con los alumnos y de reuniones preparatorias y formativas del profesorado, tan detenidas como sea necesario, para conseguir una comprensión y aceptación casi unánime. Se precisa también de un seguimiento adecuado para detectar los problemas y buscar soluciones.

En la valoración del profesorado por los alumnos (Tabla 3), estos otorgan mayor puntuación a la labor del profesor como tutor que en el conjunto de su actividad, lo cual es fácilmente explicable, por la relación más estrecha que mantuvieron con el profesor tutor. Sin embargo cuando valoran en conjunto el comportamiento de los profesores toman en consideración otras actividades como las clases teóricas en las que participaban casi todos los profesores.

$\mathrm{Al}$ finalizar el curso y tras conocer sus calificaciones, hubo variaciones en las percepciones respecto al proyecto de innovación docente, tal como se refleja cuando ciertas preguntas se repitieron en las diversas encuestas realizadas. Así recordemos que los alumnos estimaron (Tabla 2 y figura 1 ) que en el segundo cuatrimestre habían mejorado significativamente sus prácticas, la docencia teórica, y su capacidad para presentar sesiones clínicas.

Respecto a la priorización de las competencias nuestros alumnos coincidieron con la valoración del conjunto de los profesionales médicos de las encuestas de la $\mathrm{CND}^{11}$, en señalar las mismas nueve competencias generales más importantes, y sin embargo en las competencias específicas solo coincidieron en 4 de las 17 señaladas (Tabla 4). Se precisa pues, hacer una reflexión sobre esta falta de concordancia en las 
Tabla 4 Comparación de la priorización de las competencias genéricas y especificas efectuada por los alumnos y por el conjunto de profesionales médicos en la encuesta realizada por la Conferencia Nacional de Decanos.

RESPUESTAS DE LOS PROFESIONALES MEDICOS EN LA ENCUESTA DE LA CONFERENCIA NACIONAL DE DECANOS

\section{COMPETENCIAS GENERICAS}

\section{(1) INSTRUMENTALES}

1. Capacidad de análisis y síntesis

2. Toma de decisiones

3. Resolución de problemas

(2) PERSONALES

1. Compromiso ético

2. Razonamiento crítico

3. Trabajo en equipo

(3) SISTÉMICAS

1. Aprendizaje autónomo

2. Motivación por la calidad

3. Adaptación a nuevas situaciones

\section{COMPETENCIAS ESPECIFICAS}

(1) VALORES PROFESIONALES, ACTITUDES,

COMPORTAMIENTOS Y ÉTICA

1. Reconocer los elementos esenciales de la profesión médica, incluyendo los principios éticos y las responsabilidades legales.

2. Reconocer las propias limitaciones y la necesidad de mantener y actualizar su competencia profesional.

3. Comprender la importancia de tales principios para el beneficio

del paciente, de la sociedad y la profesión, con especial atención

al secreto profesional

\section{(2) FUNDAMENTOS CIENTÍFICOS DE LA MEDICINA}

1. Comprender y reconocer los efectos, mecanismos y manifestaciones de la enfermedad sobre la estructura y función del cuerpo humano.

2. Comprender, los fundamentos de acción, indicaciones y eficacia de

las intervenciones terapéuticas, basándose en la evidencia científica disponible.

3. Comprender y reconocer la estructura y función normal del cuerpo humano,

a nivel molecular, celular, tisular, orgánico y de sistemas, en las distintas etapas de la vida.

\section{(3) HABILIDADES CLÍNICAS}

1. Obtener y elaborar una historia clínica que contenga toda la información relevante.

2. Tener capacidad para elaborar un juicio diagnóstico inicial

y establecer una estrategia diagnóstica razonada.

3. Reconocer y tratar las situaciones que ponen la vida

en peligro inmediato, y aquellas otras que exigen atención inmediata.

(4) HABILIDADES DE COMUNICACIÓN

1. Escuchar con atención, obtener y sintetizar información pertinente acerca

de los problemas que aquejan al enfermo, y comprender el contenido

de esta información.

2. Comunicarse de modo efectivo y claro, tanto de forma oral como escrita con

los pacientes, los familiares, los medios de comunicación y otros profesionales.

(5) SALUD PÚBLICA Y SISTEMAS DE SALUD

1. Reconocer los determinantes de la salud en la población, tanto los genéticos como los dependientes del estilo de vida, demográficos, ambientales, sociales, económicos, psicológicos y culturales.

2. Asumir su papel en las acciones de prevención y protección ante enfermedades, lesiones o accidentes y mantenimiento y promoción de la salud tanto a nivel individual como comunitario.

(6) MANEJO DE LA INFORMACIÓN

1. Conocer, valorar críticamente y saber utilizar las fuentes de información clínica y biomédica para obtener, organizar, interpretar y comunicar la información científica y sanitaria.

2. Saber utilizar las tecnologías de la información y la comunicación en

las actividades clínicas, terapéuticas, preventivas y de investigación.

(7) ANÁLISIS CRÍTICO E INVESTIGACIÓN

1. Tener, en la actividad profesional, un punto de vista crítico, creativo, con escepticismo constructivo y orientado a la investigación.

2. Ser capaz de formular hipótesis, recolectar y valorar de forma crítica

la información para la resolución de problemas, siguiendo el método científico.
RESPUESTAS DE LOS ALUMNOS

\section{COMPETENCIAS GENERICAS}

(1) INSTRUMENTALES

1. Capacidad de análisis y síntesis

2. Toma de decisiones

3. Resolución de problemas

(2) PERSONALES

1. Razonamiento crítico

2. Compromiso ético

3. Trabajo en equipo

(3) SISTÉMICAS

1. Motivación por la calidad

2. Adaptación a nuevas situaciones

3. Aprendizaje autónomo

\section{COMPETENCIAS ESPECIFICAS}

(1) VALORES PROFESIONALES, ACTITUDES, COMPORTAMIENTOS Y ÉTICA

1. Desarrollar la práctica profesional con respeto a otros profesionales de la salud.

2. Saber aplicar el principio de justicia social a la practica profesional

3. Reconocer las propias limitaciones y la necesidad de mantener

y actualizar su competencia profesional.

(2) FUNDAMENTOS CIENTÍFICOS DE LA MEDICINA

1. Comprender y reconocer los efectos del crecimiento, el desarrollo y el envejecimiento sobre el individuo y su entorno social.

2. Comprender, los fundamentos de acción, indicaciones y eficacia de

las intervenciones terapéuticas, basándose en la evidencia científica disponible.

3. Reconocer las bases de la conducta humana normal y sus alteraciones.

(3) HABILIDADES CLÍNICAS

1. Indicar la terapéutica más adecuada de los procesos agudos y crónicos más prevalentes, así como de los enfermos en fase terminal.

2. Plantear y proponer las medidas preventivas adecuadas a cada situación clínica.

3. Establecer el diagnóstico, pronóstico y tratamiento aplicando los principios basados en la mejor información posible.

(4) HABILIDADES DE COMUNICACIÓN

1. Redactar historias clínicas y otros registros médicos de forma comprensible a terceros.

2. Establecer una buena comunicación interpersonal, que capacite para dirigirse con eficiencia y empatía a los pacientes, a los familiares, medios de comunicación y otros profesionales.

(5) SALUD PÚBLICA Y SISTEMAS DE SALUD

1. Obtener y utilizar datos epidemiológicos y valorar tendencias y riesgos para la toma de decisiones sobre salud.

2. Reconocer su papel en equipos multiprofesionales, asumiendo el liderazgo cuando sea apropiado, tanto para el suministro de cuidados de la salud, como en las intervenciones para la promoción de la salud.

(6) MANEJO DE LA INFORMACIÓN

1. Mantener y utilizar los registros con información del paciente para

su posterior análisis, preservando la confidencialidad de los datos.

2. Saber utilizar las tecnologías de la información y la comunicación

en las actividades clínicas, terapéuticas, preventivas y de investigación.

(7) ANÁLISIS CRÍTICO E INVESTIGACIÓN

1. Ser capaz de formular hipótesis, recolectar y valorar de forma crítica la información para la resolución de problemas, siguiendo el método científico.

2. Comprender la importancia y las limitaciones del pensamiento científico

en el estudio, la prevención y el manejo de las enfermedades. 
competencias específicas para la modificación de los futuros planes de estudios. Con el transcurso del proyecto se observaron algunas diferencias significativas en las respuestas de los alumnos de forma que, para los valores profesionales disminuyó el porcentaje de la aplicación de la justicia social y se incrementó para el hecho de reconocer las propias limitaciones; también se observó que respecto a las habilidades clínicas se incrementó significativamente la elección de indicar la terapéutica mas adecuada (Figura 1). En el análisis comparativo de los resultados de la encuesta sobre competencias del Anexo I del Libro Blanco del titulo de Grado de Medicina de la ANECA $^{11}$, se había observado que existían muchas más coincidencias entre las opiniones de los gestores sanitarios y de los graduados, que entre las opiniones de éstos dos grupos y las de los profesores. En general, todas las destrezas interpersonales tendieron a ser consideradas menos importantes por los profesores universitarios que por los graduados y los gestores sanitarios ${ }^{21}$.

La estimación de horas a la semana que precisan los alumnos para realizar las tareas derivadas de las prácticas y para la preparación de los casos clínicos posiblemente haya sido subestimada por los profesores (28.75 horas), pero tampoco parece consistente la estimación de los alumnos pues para las 5 asignaturas el promedio esta era de 75.6 horas a la semana, cuando no debería exceder de 72 horas si para los cálculos tenemos en cuenta que los días de docencia el horario de mañana es de 8 a 15, y que por la tarde tienen como promedio 1-2 horas para las asignaturas optativas y de libre elección, a lo que habría que sumar un mínimo de 7 horas para dormir, y los fines de semana podrían estudiar un máximo (excepcional) de 16 horas al día.

La estimación de las horas a la semana que precisan los alumnos para la preparación de los exámenes fue subestimada por los profesores ( 28 horas), pero tampoco parece consistente la estimación de los alumnos. Así para la asignatura de Pediatría las clases terminaron el día 11 de Junio y el examen fue el 16 de Junio (fecha fijada por los alumnos), por lo que sí se estima que durante la última semana de clase estudiaban Pediatría 12 horas al día sería 60 horas, y si se consideran 17 horas al día durante los últimos 4 días (periodo lectivo sin docencia) obtendríamos un valor máximo en torno a las 128 horas, cifra muy inferior a la estimada por los alumnos de 196.7 horas. Posiblemente la estimación de horas debería hacerse promediando las de profesores y alumnos, aunque nuestros alumnos tienden a estudiar poco a lo largo del curso y concentran las horas de estudio en los periodos de exámenes, por lo que se precisa un cambio en el planteamiento del estudio desde los primeros cursos de la carrera.

Nosotros habíamos utilizado el método impositivo ${ }^{13,15}$ para la asignación de horas a las diferentes actividades que deben desarrollar los alumnos, de forma similar a lo propuesto por otras Universidades ${ }^{22}$, si bien se precisan de trabajos experimentales como el nuestro para ajustar mejor las cargas de trabajo de los alumnos, independientemente de que se puedan utilizar en el futuro otros métodos de asignación como el compositivo o el de asignación de créditos de acuerdo con los resultados del aprendizaje ${ }^{14,15}$.

Respecto a las infraestructuras, estas han sido claramente deficientes dado que las asignaturas de segundo ciclo carecen de espacios específicos en la Facultad de Medicina y en sus dependencias sólo hay para toda la licenciatura 6 aulas grandes (una por curso) y otras 3 pequeñas para todos los cursos. Ello obligó a que tan sólo se pudieran dividir a los alumnos en un máximo de 2 grupos para los seminarios y los casos clínicos.

La formación del profesorado era la tradicional en las Facultades de Medicina, y por tanto existían necesidades formativas relacionadas con la convergencia europea, sobre todo para estimular la participación de los alumnos y para la enseñanza a pequeños grupos, así como para elaborar las herramientas necesarias para la tutorización de los alumnos y la evaluación continuada, y sería recomendable la realización de talleres formativos en dichos temas.

En cuanto al número de profesores observamos que en el área de conocimiento de Pediatría la dedicación de los profesores suma 90 horas, y dado que todos ellos debían impartir también otra asignatura en sexto curso (Clínica Pediátrica), su dedicación a la asignatura de Pediatría en quinto curso fue de 52 horas mientras que la actividad desarrollada fue de 60.5 horas a la semana, por lo que para la adaptación a éstos nuevos planes de docencia deberá incrementarse el número de profesores si se quiere mejorar la calidad de la docencia. En las asignaturas de Patología Médica las horas de docencia (teórica y práctica) impartidas por los profesores fueron de 76.5 horas a la semana, pero según los planes de organización docente el computo total de horas disponibles para dichas asignaturas era sólo de 42 horas. En Patología Quirúrgica los profesores impartían 24.5 horas a la semana, y los profesores asignados sumaban 32 horas de docencia y 24 de tutorías, pero debían impartir también otras asignaturas con un total de 62 horas en el plan de organización docente. 
Podemos concluir por tanto que la introducción del ECTS mejoró el rendimiento académico, la formación práctica y el autoaprendizaje de los alumnos. El proyecto de innovación fue satisfactorio para profesores y alumnos. La estimación de la carga de trabajo de los alumnos fue subestimada. Existen diferencias entre los alumnos y los profesionales médicos para priorizar las competencias específicas del médico. Para la convergencia europea se precisa mejorar la formación del profesorado en nuevas técnicas metodológicas docentes y sistemas de evaluación, mejorar las infraestructuras y aumentar el personal docente.

\section{AGRADECIMIENTOS}

Estudio realizado con la ayuda de una proyecto de innovación docente concedido por la Universidad de La Laguna.

Queremos agradecer la colaboración de los alumnos y de todos los profesores de las asignaturas que integran el segundo cuatrimestre de quinto curso, sin cuya participación este trabajo no hubiera sido posible.

\section{BIBLIOGRAFÍA}

1. XVII Congreso de la Sociedad Española de Educación Médica Convergencia europea en el Grado. Educ Méd. 2005; 8; 113-119

2. Declaración de la Sorbona. Joint declaration on harmonisation of the architecture or the European higher education system by the four Ministers in charge for France, Germany, Italy and the United Kingdom. Paris 25 de Mayo de 1998. Descargado de http://www.crue.org/sorbo-in.htm

3. Declaración de Bolonia. The European Higher Education Area. Joint declaration of the European Ministers of Education. Bolonia 19 de Junio de 1999. Descargado de: http://www.crue.org/decbolognaingles.htm

4. Declaración de Bergen. From Berlin to Bergen and beyond Ministers responsible for higher education in 45 European countries met in Bergen on 19-20 May 2005. Descargado de http://www.bologna-bergen2005.no/.

5. Real Decreto $1125 / 2003$, de 5 de septiembre, por el que se establece el sistema europeo de créditos y el sistema de calificaciones en las titulaciones universitarias de carácter oficial y validez en todo el terreno nacional. BOE 18 de septiembre de 2003.

6. Directiva Europea 2001 (1). 12/ 2004, 13781/2/04 REV 2, de 21 de Diciembre de 2004.

7. Real Decreto 55/2005, de 21 de enero, por el que se establece la estructura de las enseñanzas universitarias y se regulan los estudios universitarios oficiales de Grado (BOE 25 de Enero de 2005, pp 2842-2846).

8. Peinado Herreros JM. Competencias Médicas. Educ Med. 2005; 8 (Suppl. 2): S4-S6.
9. Sociedad Española de Educación Médica (SEDEM), Assosiació Catalana d'Educació Mèdica (ACEM), Sociedad de Educación Médica de Euskadi (SEMDE) y Sociedad Aragonesa de Educación Médica (SADEM). Recomendaciones para un nuevo proceso de reforma curricular en las facultades de medicina españolas. Educ méd. 2005; 8: 3-7.

10.Palés J, Vallés A, Cardellach F, Gomar C, Estrach MT y cols. Habilidades y procedimientos clínicos básicos a adquirir por los estudiantes de la Facultad de Medicina de la Universidad de Barcelona. Educ méd. 2001; 4: 72-81.

11.Conferencia Nacional de Decanos de la Facultades de Medicina. Libro Blanco Título de Grado de Medicina. ANECA.20 de Abril de 2005. 596 páginas. Descargado de http://www.aneca.es/modal_eval/conver_docs_titulos.html.

12.Global Minimum Essential Requirements in Medical Education. Institute for International Medical Education Core Committee. Med Teach 2002; 24:130-135.

13.Vicerrectorado de Planes de Estudios y Títulos propios. Recomendaciones para el programa docente de las asignaturas que participan en el Proyecto piloto de experimentación del ECTS. Curso 2004-05. 30 de Abril de 2004. 8 páginas. Descargado de http://www.ull.es/docencia/creditoeuropeo/recomendaciones_ECTS.pdf

14.Pagani R. Informe Técnico: El crédito europeo y el sistema educativo español. 2002. 42 páginas. Descargado de : www.crue.org/espaeuro/encuentros/credito.pdf.

15.Lavigne R. Créditos ECTS y métodos para su asignación. 2003. Descargado de:

www.aneca.es/modal_eval/docs/doc_conv_gral1.pdf 15 páginas.

16.Likert R. A technique for the measurement of attitudes. Arch Psychol. 1932; 140: 1-55.

17.Summerfield M, Youngman M. The relationship between personality and attainment in 16-19 year-old students in a sixth form college: Construction of the Student Self-Perception Scale. Br J Educ Psychol. 1999; 69: 159-172.

18. Maranillo E, Mirapeix RM, Reig J, Branda L. Aprendizaje basado en problemas aplicado a grupos numerosos. Educ méd. 2005; 8: 140A

19. Valero M, Aramburu J, Baños JE, Girvent M, Pérez J, Senti M. Introducción del portafolio: Dificultades y satisfacción. Educ Méd 2005; 8: 155A

20.Valero M, Albaina S, Aramburu J, Baños JE, Girvent M, Pérez J y Sentí M. Objetividad en la evaluación de un portafolio. Educ méd. 2005; 8: 168 A.

21. Carreras Barnés J. Situación actual de la convergencia europea en los estudios de Ciencias de la Salud: Competencias genéricas ¿Quién las define? ¿Cómo se adquieren? ¿Cómo se evalúan?. Educ méd 2005; 8 (Supl. 1): S9

22.Torres Cantero AM. Primera Jornada sobre Convergencia al Espacio Europeo de Educación Superior en Ciencias de la Salud. 26 de Abril de 2005. 25 páginas. Descargado de http://www.um.es/medicina/actividades/2005/ciencias-saludees/LaConvergenciaEuropeaCienciasSalud.ppt\#1 